\title{
Kang-Redner Anomaly in Cluster-Cluster Aggregation
}

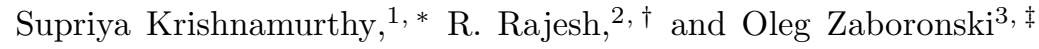 \\ ${ }^{1}$ Santa Fe Institute, 1399 Hyde Park Road, Santa Fe, NM 87501, USA \\ ${ }^{2}$ Department of Physics - Theoretical Physics, University of Oxford, 1 Keble Road, Oxford OX1 3NP, UK \\ ${ }^{3}$ Mathematics Institute, University of Warwick, Gibbet Hill Road, Coventry CV4 7AL, UK
}

(Dated: November 20, 2018)

\begin{abstract}
The large time, small mass, asymptotic behavior of the average mass distribution $\bar{P}(m, t)$ is studied in a $d$-dimensional system of diffusing aggregating particles for $1 \leq d \leq 2$. By means of both a renormalization group computation as well as a direct re-summation of leading terms in the small reaction-rate expansion of the average mass distribution, it is shown that $\bar{P}(m, t) \sim \frac{1}{t^{d}}\left(\frac{m^{1 / d}}{\sqrt{t}}\right)^{e_{K R}}$ for $m \ll t^{d / 2}$, where $e_{K R}=\epsilon+O\left(\epsilon^{2}\right)$ and $\epsilon=2-d$. In two dimensions, it is shown that $\bar{P}(m, t) \sim \frac{\ln (m) \ln (t)}{t^{2}}$ for $m \ll t / \ln (t)$. Numerical simulations in two dimensions supporting the analytical results are also presented.

PACS numbers: 05.10.Cc, 05.70.Ln, 82.40.Qt
\end{abstract}

\section{INTRODUCTION}

Reaction-diffusion systems in low dimensions provide an excellent testing ground for developing our understanding of the fluctuation effects in complex systems far from equilibrium. A great deal of information, both numerical and analytical, has been gathered over the past twenty years to show that the evolution of statistical properties in simple reaction diffusion systems in low dimensions is anomalous, in the sense that it does not follow the corresponding mean field equations (see (1] for a short review). In low enough dimensions, in the instances where an exact solution is lacking, there are no formal methods by which the exponents characterizing the different physical quantities may be calculated. The renormalization group method (see [2] for a review) provides the only systematic way to calculate these exponents and thus understand fluctuation-dominated kinetics in reaction-diffusion systems. In this paper, we consider the model of irreversible aggregation of diffusing, massive particles $A_{i}+A_{j} \stackrel{\lambda}{\rightarrow} A_{i+j}$, in dimensions $1 \leq d \leq 2$, and use the renormalization group method to calculate the small mass $\left(m \ll t^{d / 2}\right)$ behavior of $\bar{P}(m, t)$, the mean density of particles of mass $m$ at time $t$. As we explain later in this section, this problem of determining $\bar{P}(m, t)$ requires using the full power of the renormalization group method. This is unlike many other problems where just considering the rate equations with a renomalised reaction rate $\lambda$ is enough to obtain the right answer.

We now give a more precise definition of the clustercluster aggregation (CCA) model $A_{i}+A_{j} \stackrel{\lambda}{\rightarrow} A_{i+j}$ and review earlier relevant results. Consider a $d$-dimensional lattice and particles that possess a positive mass. Given

\footnotetext{
*Electronic address: supriva@santafe.edu

${ }^{\dagger}$ Electronic address: r.ravindran1@phvsics.ox.ac.uk

${ }^{\ddagger}$ Electronic address:
}

a configuration of particles on this lattice, the system evolves in time via the following microscopic moves: (i) With rate $D$, each particle hops to a nearest neighbor and (ii) with rate $\lambda$, two particles on the same lattice site aggregate together to form a new particle whose mass is the sum of masses of the two constituent particles. As time increases, the number of particles decreases due to collisions and ultimately when $t \rightarrow \infty$, all particles coagulate together to form one massive aggregate. However, at finite times there is a well defined average mass distribution $\bar{P}(m, t)$ which is of interest to determine. It will be shown later (see the text following Eq. (16)) that the large time limit of this model is the same as the large $\lambda$ limit. The $\lambda \rightarrow \infty$ limit was studied numerically by Kang and Redner in one and two dimensions [3]. It was shown that $\bar{P}(m, t)$ has the scaling form $\bar{P}(m, \vec{t})=t^{-d} f\left(m t^{-d / 2}\right)$, where $d \leq 2$ is the dimension. The two exponents are easily determined from the two conditions $\int m \bar{P}(m) d m \sim t^{0}$ (mass conservation) and $\int \bar{P}(m) d m \sim t^{-d / 2}$ (recurrence of random walks). The small mass behavior of $\bar{P}(m, t)$ can be obtained by knowing the small $x$ behavior of the scaling function $f(x)$. On the basis of numerical simulations it was conjectured in [3] that $f(x) \sim x^{(2-d) / d}$. In one dimension, the model can be solved exactly [4, 5]; it was shown that $f(x) \sim x$ or equivalently $\bar{P}(m, t) \sim m t^{-3 / 2}$ for $m \ll \sqrt{t}$. The one dimensional solution uses the property of ordering of particles on a line and is not generalizable to higher dimensions. In two dimensions, $f(x)$ was seen numerically to increase with $x$ for small $x$ [3]. Also, in two dimensions, the scaling function could be determined in the limit of fixed $x$ for $t \rightarrow \infty$, where $x=m \ln (t) / t$. In this case it was shown [6] that $\bar{P}(m, t)=t^{-2} \ln ^{2}(t) e^{-x}$ for $x \ll \ln ^{1 / 2}(t)$ and $|\ln (x)| \ll|\ln (t)|$. This result however, becomes incorrect in the limit when $m$ is fixed as $t \rightarrow \infty$.

In this paper, we compute $\bar{P}(m, t)$ in $1 \leq d \leq 2$ in the limit $t \rightarrow \infty, m / m_{0}=$ fixed, where $m_{0}$ is the mass of the lightest particle at $t=0$. We show that $\bar{P}(m, t) \sim$ 
$\frac{1}{t^{d}}\left(\frac{m^{1 / d}}{\sqrt{t}}\right)^{e_{K R}}$ for $m \ll t^{d / 2}$, where $e_{K R}=\epsilon+O\left(\epsilon^{2}\right)$ and $\epsilon=2-d$. In two dimensions, it is shown that $\bar{P}(m, t) \sim \frac{\ln (m) \ln (t)}{t^{2}}$ for $m \ll t / \ln (t)$. These results provide a theoretical basis to the results obtained by numerical methods in [3].

The CCA model may also be considered to be a special case of the more general model in which the aggregation kernel is mass dependent. For a review of results on the rate equation approach to this problem see [7, 8]. The dependence of $\bar{P}(m, t)$ on $m$ in one dimension in this more general model has also been studied [9]. In this paper, we will restrict ourselves to the aggregation kernel which is mass independent; i.e., the rates $\lambda$ and $D$ are independent of mass. When the mass is ignored and $\lambda \rightarrow \infty$, the CCA model reduces to the well studied $A+A \rightarrow A$ model [10]. The CCA model and its variants also find application in a large number of physical systems including colloidal suspensions 11, irreversible polymerisation [12], aerosols and cloud formation 12, river networks 13] and coarsening phenomena 14.

Field theoretic methods have been previously used to study complex systems far from equilibrium (see 15] for a review). We briefly review results relevant to reactiondiffusion systems. In some earlier works [16, 17], the effective reaction rate and the decay exponent of the average particle density were computed for the $A+A \rightarrow A(\emptyset)$ model. The renormalization group study of the same model with sources was done in [18]. In [19] the systematic renormalization group procedure for the computation of average density and density-density correlation function in $k A \rightarrow \emptyset$ reaction was developed. In 20] renormalization group analysis of $A+B \rightarrow \emptyset$ reaction in $d>2$ was used to study the effects of initial fluctuations on the late time decay of particle densities. The renormalization group technology developed by Peliti, Lee and Cardy [17, 19, 20] was used to compute the average mass distribution of clusters in the CCA model in the intermediate mass range in [6].

It turns out, however, that as far as the study of scaling properties of one-point correlation functions in most reaction-diffusion systems is concerned, renormalization group is not a vital tool. Consider, for example, a single species annihilation model $A+A \rightarrow \emptyset$. Once the renormalization of effective reaction rate is understood, the correct density decay exponent can be obtained from simple dimensional arguments [15]. Alternatively, one can use simple random walk arguments or use the Smoluchowski approximation (we refer to the case in which the reaction rate is replaced by a time dependent reaction rate as the Smoluchowski approximation) [3, 21, to obtain the correct values of decay exponents. A renormalized mean field theory or, alternatively, a version of Smoluchowski theory can also be used to compute the average mass distribution in cluster-cluster aggregation for intermediate masses [22].

In the CCA model considered in this paper, it turns out that the stochastic field $P(m, \vec{x}, t)$, describing the continuous limit of the local mass distribution, has a non-zero anomalous dimension in $d<2$. The scaling exponent governing the dependence of the average mass distribution $\bar{P}(m, t)$ on mass is proportional to the anomalous dimension of the operator corresponding to the local mass distribution. As explained in Section [I] any approximation scheme which disregards this anomaly (such as the Smoluchowski approximation) predicts that $\bar{P}(m, t) \sim m^{0}$ when $m \ll t^{d / 2}$, for any dimension. This is in contradiction with both numerical results [3] as well as the exact result in one dimension [4, 5. The full power of renormalization group analysis has thus to be brought to bear in order to compute the anomalous dimension of $P(m, \vec{x}, t)$ in the form of the $\epsilon=2-d$ expansion. Since the peculiarity in the small mass distribution of clustercluster aggregation is first discussed in [3] we refer to this as the Kang-Redner anomaly.

In addition to the calculation of $\bar{P}(m, t)$, we found many similarities between the problem of cluster-cluster aggregation and the problem of weak turbulence (see 23. for a review on weak turbulence). We elaborate on this connection in Sec. VI, where the Kang-Redner anomaly is interpreted as a violation of the Kolmogorov (constant flux) spectrum of particles in mass space due to strong flux fluctuations.

The rest of the paper is organized as follows. Section [1] contains a discussion of the stochastic integro differential equation satisfied by $P(m, \vec{x}, t)$. The mean field results, as well as the reasons for their failure in low dimensions are also included. In Sec. III, we analyze the large time asymptotic behavior of $\bar{P}(m, t)$ in $d<2$ using the renormalization group method. We do the same for $d=2$ in Sec. IV. In Sec. V], we rederive the results of Sec. III and IV using explicit re-summation of all diagrams giving the leading contribution to the average mass density in the limit of large time. Reasons for the failure of the Smoluchowski theory then become more transparent. In Sec. VI we elaborate on the connections with weak turbulence. Finally, we conclude with a summary and discussion of open problems in Sec. VII.

\section{CONSTANT KERNEL CLUSTER-CLUSTER AGGREGATION AND MEAN FIELD ANALYSIS.}

The problem of computing density correlation functions in $d$-dimensional stochastic processes can be reformulated as an effective equilibrium problem in $(d+1)$ dimensions with the help of Doi-Zeldovich-Ovchinnikov trick [24]. One can then attempt to solve the problem using the powerful methods of statistical field theory, in particular those based on renormalization group ideas. Starting from the lattice version of the CCA model, we would like to derive the corresponding field theory and the Langevin equation obeyed by $P(m, \vec{x}, t)$, where $P(m, \vec{x}, t) d m d V$ is the number of particles with masses in the interval $[m, m+d m]$ in the volume $d V$. It was shown in [6], that the problem of finding all correlation functions 
of the local mass distribution is equivalent to finding all moments of the following stochastic integro-differential equation (stochastic Smoluchowski equation):

$$
\begin{aligned}
\left(\frac{\partial}{\partial t}-D \Delta\right) P(m, \vec{x}, t)=\lambda P * P \\
\quad-2 \lambda N(\vec{x}, t) P(m, \vec{x}, t)+2 i \sqrt{\lambda} \xi(\vec{x}, t) P(m, \vec{x}, t),
\end{aligned}
$$

where $P * P=\int_{0}^{m} d m^{\prime} P\left(m-m^{\prime}, \vec{x}, t\right) P\left(m^{\prime}, \vec{x}, t\right)$ is a convolution term, $\xi(\vec{x}, t)$ is Gaussian white noise and $N(\vec{x}, t)=\int_{0}^{\infty} d m \vec{P}(m, \vec{x}, t)$, is the local particle density. We are interested in $\bar{P}(m, t)=\langle P(m, \vec{x}, t)\rangle$, where $\langle\ldots\rangle$ denotes averaging with respect to the noise $\xi$. If the initial number of particles of different masses at different lattice sites are independent Poisson random variables parameterized by initial average mass distribution $P_{0}(m)$, then the initial condition to be supplied with Eq. (11) is $P(\vec{x}, m, 0)=P_{0}(m)$. It is easy to check that Eq. (11) conserves the average mass density $\rho=\int m \bar{P}(m) d m$.

As particles aggregate the typical mass grows in time as $t^{d / 2}$. If we are interested in small masses, we need to consider masses smaller than the typical mass, $m \ll$ $\rho(D t)^{d / 2}$. This can be achieved by considering $m / m_{0}$ to be fixed as $t \rightarrow \infty$, where $m_{0}$ is the smallest mass at $t=0$. In this case, the first term on the right hand side of Eq. (11) is almost surely small compared to the other terms. Consequently, the small mass behavior of the local mass distribution is described by the following system of non-linear stochastic partial differential equations (SPDE):

$$
\begin{aligned}
\left(\frac{\partial}{\partial t}-D \Delta\right) P(m, \vec{x}, t) & =-2 \lambda N(\vec{x}, t) P(m, \vec{x}, t) \\
& +2 i \sqrt{\lambda} \xi(\vec{x}, t) P(m, \vec{x}, t), \\
\left(\frac{\partial}{\partial t}-D \Delta\right) N(\vec{x}, t) & =-\lambda N^{2}(\vec{x}, t) \\
& +2 i \sqrt{\lambda} \xi(\vec{x}, t) N(\vec{x}, t) .
\end{aligned}
$$

Equations (2) and (3) demonstrate an interesting connection between this model and $A+A \rightarrow A$ model. Stochastic field $P$ can be identified with $\frac{\partial N}{\partial N_{0}}$, where $N_{0}$ is the initial density. Differentiating Eq. (3) with respect to $N_{0}$, and setting $P=\frac{\partial N}{\partial N_{0}}$, we obtain Eq. (2).

We are interested in the behavior of $\bar{P}(m, t)$ in the limit of fixed $m$ and $t \rightarrow \infty$. We can then identify the particles with this fixed mass as $B$ kind of particles and the remaining particles as $A$ kind of particles. Then, clearly, the study of Eqs. (2) and (3) is equivalent to the study of a two species reaction

$$
\begin{aligned}
& A+A \stackrel{\lambda}{\rightarrow} A, \\
& A+B \stackrel{\lambda}{\rightarrow} \text { Inert },
\end{aligned}
$$

in the limit when concentration of $B$ particles is much smaller than that of $A$ particles. This two species problem has been studied in $d=1$ for arbitrary diffusion rates
[25]. Specializing results of this paper to our case, we find that $\bar{P}(m, t) \sim t^{-3 / 2}$ for $t \rightarrow \infty$. Assuming that the large time asymptotics of $\bar{P}(m, t)$ is universal, we can restore the $m$-dependence using dimensional analysis, to obtain

$$
\bar{P}(m, t)=C \frac{m}{\rho t^{3 / 2}},
$$

where $C$ is a constant, and $\rho$ is the average mass density. Equation (5) matches with the exact results obtained for the CCA model in one dimension [4.

However, no exact solutions are available for dimensions $d>1$. In the rest of the paper we will be analyzing Eqs. (2) and (3) in $d>1$ using the dynamical renormalization group method. We will show that for small masses and $1 \leq d<2$,

$$
\bar{P}(m, t) \sim \frac{1}{\rho(D t)^{d}}\left(\frac{m}{\rho(D t)^{d / 2}}\right)^{e_{K R}}, m \ll \rho(D t)^{d / 2},
$$

where $e_{K R}=\epsilon+O\left(\epsilon^{2}\right)$ and $\epsilon=2-d$. If $d=2$,

$$
\begin{aligned}
P(m, t) \sim & \frac{1}{\rho(D t)^{2}} \ln \left(t / t_{0}\right) \ln \left(\frac{m}{\rho D t_{0}}\right)\left(1+\frac{1}{\ln \left(t / t_{0}\right)}\right), \\
& \text { for } \rho D t_{0} \ll m \ll \rho D t \ln \left(t / t_{0}\right) .
\end{aligned}
$$

Here, $t_{0} \sim \Delta^{2} / D$, where $\Delta$ is the lattice spacing.

Before doing the renormalization group analysis, let us analyze Eqs. (2) and (3) in the mean field (weak coupling) limit. Neglecting stochastic terms in the right hand side of Eqs. (2) and (3) and solving the resulting system of ordinary differential equations, we obtain

$$
\begin{aligned}
& \bar{N}_{M F}(t)=\frac{N_{0}}{1+N_{0} \lambda t}, \\
& \bar{P}_{M F}(t)=\frac{P_{0}}{\left(1+N_{0} \lambda t\right)^{2}} .
\end{aligned}
$$

Thus, at large times, $\bar{P}(t) \sim t^{-2}$, given that mean field theory is applicable. Relative corrections to the mean field result are of the order $g_{0}(t)=\frac{\lambda t}{(D t)^{d / 2}}$. Therefore mean field theory is asymptotically exact in $d>2$ [26] and diverges with $t$ if $d<2$. Re-summation of the most divergent terms in the weak coupling expansion of $\bar{P}$ around the classical solution is required and can be performed in the case at hand using the formalism of renormalization group. The details of the computation are given in the next Sec. III. Here, we would like to demonstrate that accounting for renormalization of the coupling constant alone doesn't yield the correct decay exponent as mentioned earlier. To the leading order in $\epsilon=2-d$, renormalization of the effective reaction rate reduces to replacing $\lambda$ in Eqs. (2) and (3) with renormalized value $\lambda_{R}=f(\epsilon) t^{-\epsilon / 2}$ and omitting stochastic terms (renormalized mean field approximation). Eliminating $\bar{N}(t)$ from the resulting system of ordinary differential equations one finds the following equation for $\bar{P}(m, t)$ :

$$
\frac{\partial \bar{P}}{\partial t}=-d \frac{\bar{P}}{t} .
$$




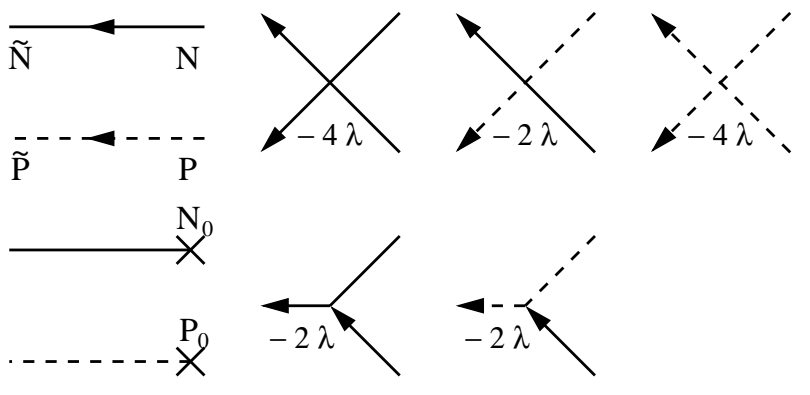

FIG. 1: Propagators and vertices of the effective theory, Eq. (12)

This implies, that $\bar{P}(m, t) \sim m^{0} t^{-d}$. In other words, $\bar{P}(m, t)$ scales with time according to its physical dimension. As a result it does not depend on mass. As we will show in Sec. III, arguments leading to this conclusion are incorrect, as they disregard the possibility of anomalous dimension of the stochastic field $P$.

\section{RENORMALIZATION GROUP ANALYSIS OF STOCHASTIC AGGREGATION.}

The average mass distribution $\bar{P}(t)$ and average particle density $\bar{N}(t)$ admit functional integral representations which can be obtained by applying the Martin-SiggiaRose procedure [27] to Eqs. (2) and (3) (equivalently see Eqs. (2)-(4) of [6]). We then obtain

$$
\begin{aligned}
\langle O(t)\rangle= & \int \mathcal{D} N\left(\vec{x}^{\prime}, t^{\prime}\right) \mathcal{D} \tilde{N}\left(\vec{x}^{\prime}, t^{\prime}\right) \mathcal{D} P\left(\vec{x}^{\prime}, t^{\prime}\right) \mathcal{D} \tilde{P}\left(\vec{x}^{\prime}, t^{\prime}\right) \\
& \times O(t) e^{-S_{e f f}[N, \tilde{N}, P, \tilde{P}]}
\end{aligned}
$$

where $O(t)=N(\vec{x}, t)$ or $P(\vec{x}, t)$ and

$$
\begin{aligned}
& S_{\mathrm{eff}}=\int_{0}^{t} d^{d} x d \tau[\tilde{N}(\dot{N}-D \Delta N)+\tilde{P}(\dot{P}-D \Delta P) \\
& \left.+\lambda\left(\tilde{N} N^{2}+2 \tilde{P} P N+\tilde{N}^{2} N^{2}+2 \tilde{N} \tilde{P} N P+\tilde{P}^{2} P^{2}\right)\right]
\end{aligned}
$$

is the effective action functional. Perturbative expansions of $\bar{N}(t), \bar{P}(m, t)$ in powers of $\lambda$ can now be obtained in the standard way, see for example [28]. Feynman rules for constructing terms of these expansions are summarized in Fig. 1. Due to the non-renormalization of the diffusion rate as well as the average mass density in the field theory Eq. ( 12), in all that follows, we use units in which $\rho=D=1$.

The average mass distribution $\bar{P}(m, t)$ is formally given by the sum of all diagrams built out of blocks presented in Fig. 1 1 with one outgoing punctuated line ( $P$-line). The contribution from each diagram is a function of $\lambda N_{0} t$ and 'bare' dimensionless reaction rate $g_{0}(t)=\frac{\lambda t}{t^{d / 2}}$. Unless we are interested in the small time expansion of $\bar{P}(m, t)$, $\lambda N_{0} t$ cannot be treated as a small parameter. Therefore,
(A)

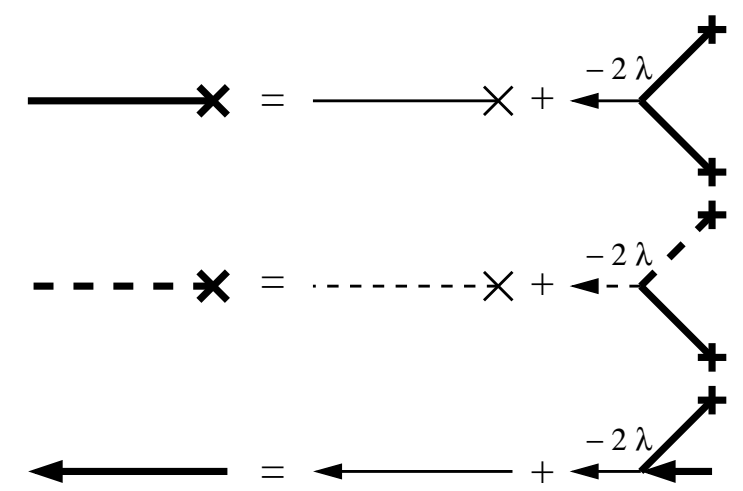

(D)

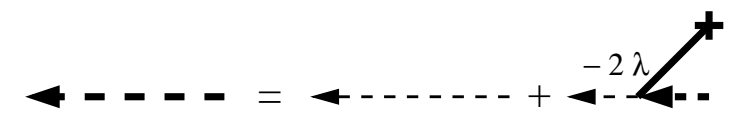

FIG. 2: Diagrammatic form of mean field equations for: (A) Average particle density; (B) Average mass distribution; (C) $N N$-response function; (D) $P P$-response function.

the contributions of all diagrams proportional to a given power of $g_{0}(t)$ and various powers of $\lambda N_{0} t$ have to be summed up. A simple combinatorial argument (see 19] for details) shows that the contribution of a diagram with $n$ loops is proportional to $g_{0}(t)^{n}$. In the weak coupling regime the main contribution to $\bar{P}(m, t)$ and $\bar{N}(t)$ is, therefore, given by the sum of all tree diagrams, the first correction comes from summing all one-loop diagrams and so on. It turns out 19,29 that the sum of all tree diagrams gives the mean field answers Eqs. (8) and (9). The expansion in powers of $g_{0}(t)$ is therefore the standard loop expansion around solutions of mean field equations. It is obvious that in $d<2$ the loop expansion is not very useful at large times as $\lim _{t \rightarrow \infty} g_{0}(t)=\infty$. Fortunately, the value of dimensionless reaction rate properly renormalized to account for the build-up of inter particle correlations turns out to be of order $\epsilon=2-d$ for large times. This allows one to convert the loop expansion into an $\epsilon$-expansion using perturbative renormalization group method. Such an expansion works well for large times and will therefore yield all the information we need about the behavior of the average mass distribution in the strongly fluctuating regime.

In computing loop corrections to any order, there are generally an infinite number of diagrams to sum. However these diagrams can be resummed in part if one introduces classical response functions $G_{c l}^{N N}$ and $G_{c l}^{P P}$. Response function $G_{c l}^{N N}\left(G_{c l}^{P P}\right)$ is equal to the sum of all tree diagrams with one outgoing and one ingoing line of types $\mathrm{N}(\mathrm{P})$. As was already mentioned, mean field densities Eqs. (8) and (9) are also equal to the sums of tree diagrams with one outgoing $\mathrm{N}$ or $\mathrm{P}$ line correspondingly. One then simply has to associate incoming lines with mean field densities and propagator lines with mean field response functions. Integral equations satisfied by classical densities and response functions are presented 


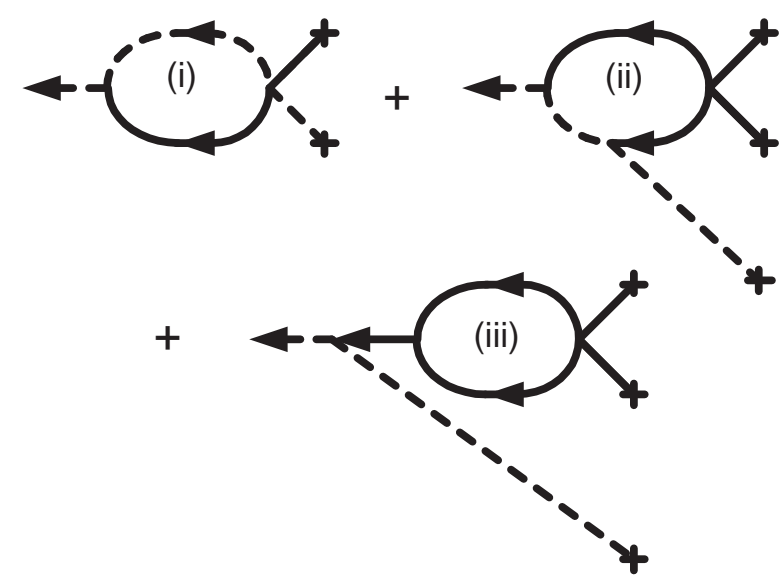

FIG. 3: One-loop corrections to the mean field answer for average mass distribution.

in diagrammatic form on Fig. 2.

Solutions of Eqs. Fig. 2(A) and Fig. 2(B) coincide with Eqs. (8) and (9), as they should. Equations Fig. 2(C) and Fig. 2(D) can also be solved with the result

$$
\begin{array}{r}
G_{c l}^{N N}\left(x_{2}, t_{2} ; x_{1}, t_{1}\right)=G_{c l}^{P P}\left(x_{2}, t_{2} ; x_{1}, t_{1}\right)= \\
\left(\frac{N_{0}\left(t_{2}\right)}{N_{0}\left(t_{1}\right)}\right)^{2} G_{0}\left(x_{2}-x_{1}, t_{2}-t_{1}\right),
\end{array}
$$

where $G_{0}$ is the Green's function of the linear diffusion equation.

Using the notion of mean field response functions and densities one can easily classify all one-loop diagrams contributing to average mass distribution. The result is presented in Fig. 3. A quick check shows that analytical expressions corresponding to diagrams Fig. 3(i) and Fig. 3(iii) containing primitive loops diverge in $d \geq 2$, which is consistent with that fact that upper critical dimension of the effective theory Eq. (12) is two. Computing the relevant integrals in $d=2-\epsilon$ dimensions we find the following one-loop expression for the average mass distribution:

$$
\begin{gathered}
\bar{P}(t)=\frac{P_{0}}{N_{0}^{2}}\left(\left(\frac{1}{\lambda t}\right)^{2}+\frac{1}{(8 \pi)^{d / 2}} \frac{1}{\lambda t^{(2-\epsilon / 2)}} F(\epsilon)\right) \\
\times\left(1+O\left(\frac{1}{\lambda N_{0} t}\right)\right)+2 \text {-loop corrections, }
\end{gathered}
$$

where $F(\epsilon)=\frac{4}{\epsilon} \frac{1-\epsilon / 2}{(1+\epsilon / 2)^{2}(1+\epsilon / 4)}$.

Equation (14) can be used to determine the large time asymptotics of $P(t)$ in the following way. The exact average mass distribution satisfies the Callan-Symanzik equation which we will derive below. Coefficients of this equation depend on the law of renormalization of all relevant couplings of the theory Eq. (12). One relevant coupling is the effective reaction rate. Its renormalization is known [17]. Below we will show that the only other relevant coupling is the initial mass distribution $P_{0}$. We will determine it's renormalization law with one-loop precision demanding that, the expression Eq. (14) be non-singular in the limit $\epsilon \rightarrow 0$ when expressed in terms of renormalized coupling constant and renormalized initial mass distribution. This will determine coefficients of CallanSymanzik equation up to terms of order $g_{R}$, where $g_{R}$ is the renormalized dimensionless reaction rate. Solving this equation we will be able to compute the decay exponent of $\bar{P}(t)$ up to terms of order $\epsilon$.

Dimensional analysis of effective vertex parts of the theory Eq. (12) shows that the only relevant bulk coupling is the effective reaction rate. Its relevance is due to the recurrence of random walks in $d \leq 2$. Reaction rate renormalization accounts for all fluctuation effects in the $A+A \rightarrow A$ model.

However, the CCA model is more complicated and interesting due to the presence of boundary-relevant couplings. To identify them, we use the following version of dimensional analysis. Boundary coupling constants correspond to vertices with no incoming lines. As PPinteractions can be neglected in the problem at hand, we are interested in boundary vertices with at most one $P$-line. Assume for simplicity that $d=2$ (critical dimension). Assume also that the initial density $N_{0}=\infty$. This assumption is justified if one is interested in the large time behavior of correlation functions in aggregation, as $N_{0}$ flows to infinity under renormalization group transformation to increasingly larger time scales, see [15] for more details. Let $\Gamma_{\alpha, \beta}(t)$, where $\alpha=0,1 ; \beta=0,1,2, \ldots$ be the simultaneous Green's function of the theory Eq. (12) with $\alpha P$-lines and $\beta \mathrm{N}$-lines with all external momenta equal to 0. Using Eq. (13), one can express $G_{\alpha, \beta}(t)$ in terms of corresponding vertex part $V_{\alpha, \beta}(t)$ as follows:

$$
G_{\alpha, \beta}=\left(\frac{1}{t^{2}}\right)^{\alpha+\beta} \int_{0}^{t} d \tau \tau^{2(\alpha+\beta)} V_{\alpha, \beta}(\tau) .
$$

As physical dimension of $G_{\alpha, \beta}(t)$ is $(\text { Length })^{-2 \beta-4 \alpha}$, $V_{\alpha, \beta}(t) \sim t^{-\beta-2 \alpha-1}$. As a result, Eq. (15) converges at small times for any $\alpha$ if $\beta>0$. If $\beta=0$ and $\alpha=1$, Eq. (15) diverges logarithmically. This divergence can be regularised using a small-time cut-off $\frac{1}{\lambda N_{0}}$ and leads to the renormalization of the initial mass distribution $P_{0}$. The latter plays a role of the coupling associated with $V_{1,0}$.

As a result of the discussed divergence, diagrams involving $V_{1,0}$ grow with time faster compared with diagrams with the same number of loops but with no subdiagrams contributing to renormalization of $P_{0}$. Consequently, diagrams belonging to the former class have to be re-summed exactly in order to obtain the correct large time behavior of the average mass distribution.

We conclude that fluctuations in stochastic aggregation lead to two effects: renormalization of the effective reaction rate and renormalization of the initial mass distribution. It follows that renormalization of these two couplings regularizes the perturbative expansion of $\bar{P}(m, t)$ to all orders.

As it turns out, the renormalization of $P_{0}$ is solely responsible for Kang-Redner anomaly. In Sec. V we will 
analyze initial density renormalization by explicitly resumming small time divergences in the perturbative expansion for $\bar{P}(m, t)$. Now, we will show how it appears formally within the framework of perturbative renormalization group method. We follow dynamical renormalization group procedure described in [15], part I. For a review of results concerning coupling constant renormalization, see ibid, part II.

Let us fix a reference time $t_{0}>0$. Expression ( 14) evaluated at $t_{0}$ is to be made finite by absorbing the divergences appearing as $\epsilon \rightarrow 0$ into the renormalization of the reaction rate and the initial density.

Let $g_{0}=\lambda t_{0}^{\epsilon / 2}$ be the dimensionless 'bare' reaction rate. As has been shown in [17], renormalized reaction rate $g_{R}$ is related to $g_{0}$ via the following exact formula:

$$
g_{R}=\frac{g_{0}}{1+\frac{g_{0}}{g^{*}}} .
$$

Here $g^{*}=2 \pi \epsilon(1+O(\epsilon))$ is the non-trivial fixed point of the renormalization group flow in the space of effective couplings of Eq. (12). Recall that $g_{0} \sim \lambda$. Hence $\lim _{\lambda \rightarrow \infty} g_{R}=g^{*}$. It follows from the Callan-Symanzik equation that large time behavior of the $\bar{P}(m, t)$ is also determined by the fixed point value of the effective reaction rate $g^{*}$. We therefore conclude that the limits $t>0, \lambda \rightarrow \infty$ and $\lambda>0, t \rightarrow \infty$ of Kang-Redner model belong to the same universality class as claimed in the introduction.

Solving Eq. (16) with respect to $g_{0}$, expanding the result in powers of $g_{R}$ and substituting the expansion into Eq. (14) we obtain the average mass distribution at time $t_{0}$ as a power series in $g_{R}$ :

$$
\bar{P}\left(t_{0}\right)=\frac{P_{0}}{N_{0}^{2} g_{R}^{2} t_{0}^{d}}\left[1-\frac{1}{g^{*}}\left(1+O\left(g^{*}\right)\right) g_{R}+O\left(g_{R}^{2}\right)\right] .
$$

As expected, the order- $g_{R}$ term in Eq. (17) is still singular at $\epsilon=0$. To cancel the remaining divergences we have to introduce renormalized initial mass distribution $P_{R}$ :

$$
P_{R}=Z\left(g_{R}, t_{0}, \epsilon\right) P_{0},
$$

where $Z$ is a power series in $g_{R}$ with coefficients chosen in such a way, that the average mass density

$$
\bar{P}\left(t, g_{R}, P_{R}, t_{0}\right)=Z\left(g_{R}, t_{0}, \epsilon\right) \bar{P}\left(t, \lambda_{0}, P_{0}, \epsilon\right),
$$

when expressed in terms of $P_{R}, g_{R}$, is non-singular at $\epsilon=0$. Substituting Eq. (17) into Eq. (19) we find that

$$
Z=1+\frac{g_{R}}{g^{*}}+O\left(g_{R}^{2}\right)
$$

in order for $\bar{P}(t)$ to be non-singular at 1-loop level.

Now we can derive the Callan-Symanzik equation. The fact that $\bar{P}\left(t, \lambda_{0}, P_{0}, \epsilon\right)$ does not depend on the reference time $t_{0}$ leads to the following equation for $\bar{P}\left(P_{R}\right)$ :

$$
t_{0} \frac{\partial}{\partial t_{0}}\left(Z^{-1} \bar{P}\left(P_{R}\right)\right)=0 .
$$

Noticing that $\bar{P}(t)=t_{0}^{-d} \frac{P_{R}}{N_{0}^{2}} \Phi\left(t / t_{0}, g_{R}\right)$, where $\Phi$ is a dimensionless function, one can convert the above condition into Callan-Symanzik equation for $\bar{P}(t)$ :

$$
\left(t \frac{\partial}{\partial t}+\frac{1}{2} \beta\left(g_{R}\right) \frac{\partial}{\partial g_{R}}+d-\frac{1}{2} \gamma\left(g_{R}\right)\right) \bar{P}\left(t, g_{R}, P_{R}, t_{0}\right)=0,
$$

where $\beta\left(g_{R}\right)=-2 \frac{\partial}{\partial t_{0}} g_{R}$ is the beta function of the theory Eq. (12) and

$$
\gamma\left(g_{R}\right)=-2 \frac{1}{Z} t_{0} \frac{\partial}{\partial t_{0}} Z=-\frac{g_{R}}{2 \pi}+O\left(g_{R}^{2}, g_{R} \epsilon\right),
$$

is the gamma function.

It is well known, that at large times and in $d<d_{c}=2$, solutions to Eq. (21) are governed by non-trivial fixed points (zeroes) of beta-function. Differentiating Eq. (16) with respect to $t_{0}$, one finds that $\beta\left(g_{R}\right)=g_{R}\left(g_{R}-g^{*}\right)$. Hence, $\beta\left(g_{R}\right)$ has a unique non-trivial fixed point $g_{R}=$ $g^{*}$. It follows from Callan-Symanzik equation (Eq. (21)) that $\bar{P}(t) \sim t^{-d^{*}}, t \rightarrow \infty$, where

$$
d^{*}=d-\frac{1}{2} \gamma\left(g^{*}\right) .
$$

We see that scaling dimension of $\bar{P}(m, t)$ differs from it's physical dimension by a term proportional to the value of $\gamma$-function at the fixed point. This term is called the anomalous dimension of the field $P$. The physical reason for the presence of anomalous dimension is the renormalization of the initial mass distribution by small time fluctuations.

As $g^{*} \sim \epsilon$, the substitution of $g_{R}$ expansion of $\gamma$ into Eq. (23) yields the $\epsilon$-expansion for $d^{*}$. Using Eq. (22) we find that

$$
d^{*}=d+\frac{1}{2} \epsilon+O\left(\epsilon^{2}\right) .
$$

Finally, one can restore the $m$-dependence of $\bar{P}(m, t)$ using dimensional argument. The result is

$$
\bar{P}(m, t)=A(\epsilon) \frac{1}{t^{d}}\left(\frac{m^{\frac{1}{d}}}{\sqrt{t}}\right)^{e_{K R}},
$$

where $e_{K R}=\epsilon+O\left(\epsilon^{2}\right)$ is equal to twice the anomalous dimension of the stochastic field $P$.

Note that in $d=1, e_{K R}=1$ and $\bar{P}(m, t) \sim m^{1}$, which coincides with the exact answer, 㭡. However, at the moment we do not have reasons to believe, that higher order $\epsilon$-corrections to our answer for $e_{K R}$ vanish identically for any $0<\epsilon \leq 1$. Equation (24) implies that anomalous dimension of $P$ vanishes at $d_{c}=2$. Yet, it follows from general theory of perturbative renormalization group that traces of anomalous dependence of $P(m, t)$ on mass in $d<2$ must be present at the critical dimension as well. We analyze Kang-Redner anomaly in $d=2$ in Sec. IV and compare renormalization group predictions with conclusions of direct numerical simulations of the system of diffusing-aggregating point particles on the two-dimensional lattice. 


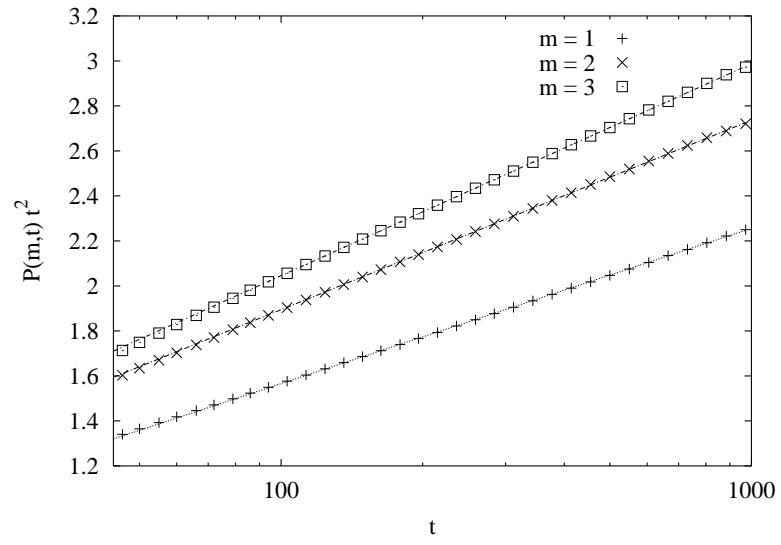

FIG. 4: The variation of $\bar{P}(m, t) t^{2}$ with $t$ is shown for $m=1,2$ and 4 on a semi-log scale. The variation is linear implying that $\bar{P}(m, t)=c(m) \ln (t) / t^{2}$, where $c(m)$ is some mass dependent function.

\section{KANG-REDNER ANOMALY IN TWO DIMENSIONS.}

It is well known that anomalies in $d_{c}-\epsilon$ dimensions lead to logarithmic corrections to mean field theory answers in $d=d_{c}$. Kang-Redner anomaly is not an exception. Small mass behavior of the average mass distribution in two dimensions can be easily obtained by solving the CallanSymanzik equation (see Eq. (21)). Note that

$$
\begin{aligned}
& \left.\beta(g)\right|_{d=2}=\frac{1}{2 \pi} g^{2}, \\
& \left.\gamma(g)\right|_{d=2}=-\frac{1}{2 \pi} g\left(1+O\left(g^{2}\right)\right) .
\end{aligned}
$$

Solving Eq. (21) with coefficients given by Eq. (26) and the initial condition

$$
\bar{P}\left(t_{0}\right)=\text { Const } \frac{1}{\mathrm{~g}_{\mathrm{R}}^{2} \mathrm{t}_{0}^{2}}\left(1+\mathrm{O}\left(\mathrm{g}_{\mathrm{R}}\right)\right),
$$

produced by the mean field theory, we find that

$$
\bar{P}(t)=\text { Const } \frac{\ln \left(\mathrm{t} / \mathrm{t}_{0}\right)}{\mathrm{t}^{2}}\left(1+\mathrm{O}\left(1 / \ln \left(\mathrm{t} / \mathrm{t}_{0}\right)\right) .\right.
$$

Such a behavior of $\left.\bar{P}(m, t)\right|_{m=\text { fixed }}$ in two dimensions was originally seen by Kang and Redner in numerical simulations $[3$. We have now shown that Eq. (28) can be obtained as a result of systematic renormalization group computation.

Analyzing their model in two dimensions, Kang and Redner noticed that $\bar{P}(m, t)$ is a slowly varying (increasing, as suggested by Fig. 2 of [3]) function of mass. We can now quantify this observation by computing the mass dependence of the average mass distribution. In $d=d_{c}$ direct dimensional arguments cannot be used to restore the mass dependence of Eq. (28) due to the explicit dependence of $\bar{P}(m, t)$ on the lattice cut-off. Instead let us analyze Eq. (25) for $\bar{P}(m, t)$ in the limit $\epsilon \rightarrow+0$. Near

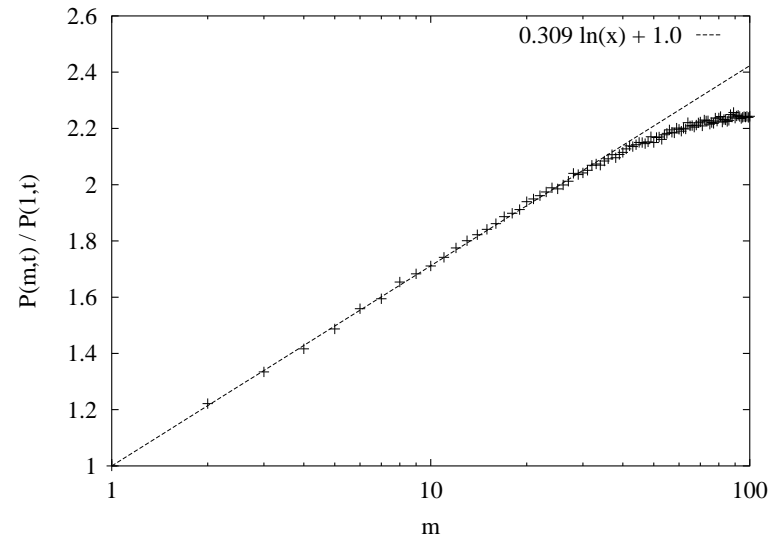

FIG. 5: The small $m$ behavior of $\bar{P}(m, t) / \bar{P}(1, t)$ is shown on a semi-log plot. The variation is linear implying that $c(m) \sim$ $\ln (m)$ with $c(m)$ as defined in caption of Fig. 4.

$\epsilon=0$, the amplitude $A(\epsilon) \sim \epsilon^{-2}$. Expanding the right hand side of Eq. (25) in powers of $\epsilon$ and using the fact that $\bar{P}(m, t ; \epsilon)$ is non-singular at $\epsilon=0$ as a function of renormalized parameters, we find that

$$
\begin{aligned}
\bar{P}(m, t)= & \frac{1}{t^{2}}\left(C_{1}\left(\ln \left(t / t_{0}\right)\right)^{2}+C_{2} \ln \left(t / t_{0}\right) \ln \left(m / t_{0}\right)\right. \\
& \left.+C_{3}\left(\ln \left(m / t_{0}\right)\right)^{2}\right)\left(1+O\left(1 / \ln \left(t / t_{0}\right)\right)\right) .
\end{aligned}
$$

We are interested in $\bar{P}(m, t)$ in the limit $t \rightarrow \infty, m=$ fixed. Time dependence of $\bar{P}(m, t)$ is given by Eq. (28). Therefore, coefficient $C_{1}$ in Eq. (29) is 0 . Hence

$$
\bar{P}(m, t)=\text { Const } \frac{\ln \left(\mathrm{t} / \mathrm{t}_{0}\right) \ln \left(\mathrm{m} / \mathrm{t}_{0}\right)}{\mathrm{t}^{2}}\left(1+\mathrm{O}\left(1 / \ln \left(\mathrm{t} / \mathrm{t}_{0}\right)\right)\right) .
$$

Note that Eq. (30) is valid for $m \ll M(t)$, where $M(t) \sim t / \ln \left(t / t_{0}\right)$ is the typical mass. If $m \sim M(t)$, then $\bar{P}(m, t) \sim\left(\ln \left(t / t_{0}\right) / t\right)^{2}$, which coincides with the answer for $\left.\bar{P}(m, t)\right|_{m \sim M(t)}$ in the intermediate mass range [6].

To check our analytical results Eq. (28) and Eq. (30) we did a numerical simulation of the system on a twodimensional lattice. For the sake of computational effeciency, we chose to work in the limit $\lambda \rightarrow \infty$ so that a lattice site can hold utmost one particle. The lattice was chosen to be a square lattice of size $3000 \times 3000$ with periodic boundary conditions. In Fig. A, we show the variation of $\bar{P}(m, t)$ with $t$ for fixed small $m$. It is seen that $\bar{P}(m, t)=c(m) \ln (t) / t^{2}$, where $c(m)$ is some function of the mass $m$. This is in excellent agreement with Eq. (28). To determine $c(m)$, we studied the variation of $\bar{P}(m, t) / \bar{P}(1, t)$ with the mass $m$ (see Fig. 5 ). We see that $c(m) \sim \ln (m)$, thus confirming Eq. (30). 


\section{KANG-REDNER ANOMALY VIA EXPLICIT RESUMMATION OF BOUNDARY DIVERGENCES}

In this section we will derive Eq. (25) without using the formalism of renormalization group. Instead, we will identify the principal set of diagrams contributing to the the large time limit of $\bar{P}(m, t)$ and derive a simple integral equation satisfied by the sum of these diagrams.

Additional divergences in the terms of the perturbative expansion of the average mass distribution discussed in the previous Sec. IV are actually due to the quadratic singularity of the mean field expression Eq. (9) for the average mass distribution at $N_{0}=\infty, t=0$. Let us illustrate this statement on the example of diagram (i) of Fig. 3. Assuming for simplicity that $d=2$ and denoting ultraviolet cut-off by $\Delta t$, we find that the most divergent contribution coming from this diagram is

$I_{(i)} \sim \frac{P_{0}}{2 \pi \lambda^{2} N_{0}^{2} t^{2}} \ln \left(\frac{t}{\Delta t}\right) \int_{0}^{t} d \tau \frac{\lambda N_{0}}{1+\lambda N_{0} \tau}\left(1+O\left(1 / \lambda N_{0} t\right)\right)$.

Setting $N_{0}=\infty$ under the sign of integration is clearly impossible, as the resulting integral would diverge. Direct computation of Eq. (31) shows that

$$
I_{(i)} \sim \frac{P_{0}}{2 \pi \lambda^{2} N_{0}^{2} t^{2}} \ln \left(\frac{t}{\Delta t}\right) \ln \left(\lambda N_{0} t\right)\left(1+O\left(1 / \lambda N_{0} t\right)\right) .
$$

We see that $I_{(i)}$ is proportional to $\ln (t)^{2}$, not $\ln (t)$ as one would have deduced from the simple loop counting. The additional singularity is due to the fact that $\left.\bar{P}_{M F}\right|_{N_{0}=\infty} \sim t^{-2}$. (Recall that $\left.\bar{N}_{M F}\right|_{N_{0}=\infty} \sim t^{-1}$. As a result divergences at $t=0$ are absent in the field theory of $A+A \rightarrow 0$ reaction.) The additional boundary singular terms are generally present in all Feynman integrals contributing to $\bar{P}(m, t)$ and have to be re-summed to all orders of perturbation theory to get the correct large time asymptotics of the average mass distribution.

Let $\Pi\left(t_{2}, t_{1}\right)$ be the exact zero momentum vertex function of type $\tilde{P} P$ - the sum of all one-particle irreducible diagrams contributing to $\tilde{P} P$ response function divided by the propagators corresponding to external lines. The Schwinger-Dyson equation for the exact average mass distribution reads:

$$
\bar{P}(t)=\bar{P}_{M F}(t)+\frac{1}{t^{2}} \int_{0}^{t} d t_{2} \int_{0}^{t_{2}} d t_{1} \Pi\left(t_{2}, t_{1}\right) \bar{P}\left(t_{1}\right) .
$$

Equation (33) is most easily derived using the formalism of Feynman diagrams. Its diagrammatic representation is given in Fig. 6 (I).

We know that in two dimensions $\bar{P}(t) \sim t^{-2}$. Therefore, near $d=2$, the function $\eta(t)=\bar{P}(t) \lambda t^{2}$ is slowly varying. The following simplified version of Eq. (33) is therefore valid to the leading order in $\epsilon$ :

$$
\eta(t)=1+\int_{0}^{t} d t_{1} \Pi\left(t_{1}\right) \eta\left(t_{1}\right)
$$
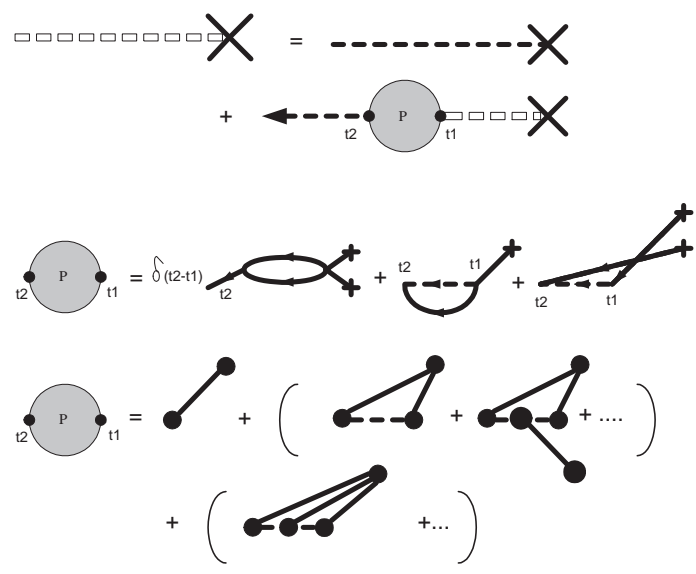

FIG. 6: (I) Schwinger-Dyson Equation for $\Pi(t)$. (II) Perturbative expansion of the polarization operator: (IIA)- in the number of loops; (IIB)- in the order of exact vertex parts.

where

$$
\Pi(t)=t^{2} \int_{0}^{t} \frac{d t_{1}}{t_{1}^{2}} \Pi\left(t, t_{1}\right) .
$$

Differentiating Eq. (34) with respect to time, we find that $\eta(t)$ satisfies the following differential equation:

$$
\frac{d \eta}{d t}(t)=\Pi(t) \eta(t)
$$

It turns out (see below), that at large times

$$
\Pi(t)=\frac{1}{t}\left(\frac{\epsilon}{2}+O\left(\epsilon^{2}\right)\right)\left(1+O\left(1 / t^{\epsilon / 2}\right)\right) .
$$

Solving Eq. (36) with $\Pi(t)$ given by Eq. (37), we find that $\bar{P}(t) \equiv \eta(t) / t^{2} \sim t^{\left(-2+\epsilon / 2+O\left(\epsilon^{2}\right)\right)}$. Thus, we have been able to rederive Eq. (24) without any reference to renormalization group.

Before we turn to the derivation of Eq. (37), we would like to demonstrate, that solving integral equation Eq. (34) is indeed equivalent to summing leading boundary singular terms in the perturbative expansion of $\bar{P}(t)$ to all orders. All divergences at $t=0, N_{0}=\infty$ can be regularised by setting the lower limit of integration in the integral in the right hand side of Eq. (34) equal to $\frac{1}{\lambda N_{0}}$. Resulting equation,

$$
\eta(t)=1+\frac{\epsilon}{2} \int_{\frac{1}{\lambda N_{0} t}}^{t} \frac{d t_{1}}{t_{1}} \eta\left(t_{1}\right),
$$

can be solved using the method of consecutive approximations. Setting $\eta(t)=\eta_{0}(t)+\eta_{1}(t)+\eta_{2}(t)+\ldots$ and treating the integral in the right hand side of Eq. (38) perturbatively, we find that $\eta_{0}(t)=1$ and

$$
\eta_{n}(t)=\frac{1}{n !}\left(\frac{\epsilon}{2} \ln \left(\lambda N_{0} t\right)\right)^{n}, \quad n=1,2, \ldots
$$


Note that $\eta_{1}(t)$ is equal to 1-loop boundary singular term, $\eta_{2}(t)$ - 2-loop boundary singular term and so on. Summing the resulting series for $\eta$ one confirms our main result that $\eta(t) \sim t^{\epsilon / 2}$. One loop-boundary singularity leads to mass-dependent logarithmic corrections to the average mass distribution. This is most easily seen by expanding Eq. (25) in powers of $\epsilon$. These corrections, which were originally observed in [6], motivated to a certain extent the present investigation.

The knowledge of the first term in the $\epsilon$-expansion of decay exponent of $\eta(t)$ leads to a good approximation of the average mass distribution, given that $\epsilon \ln \left(\lambda N_{0} t\right) \sim$ 1 , but $\epsilon^{2} \ln \left(\lambda N_{0} t\right) \ll 1$. If the latter condition breaks down, we need to know the expansion of the polarization operator $\Pi(t)$ to second order in $\epsilon$ and modify Eq. (34) by including corrections proportional to the derivative $\eta(t)$ (which account for the fact that $\eta(t)$ is not constant).

We will now derive Eq. (37) by computing $\Pi(t)$ to the first order in $\epsilon$ using 1-loop diagrams and verifying that higher-loop diagrams lead to terms of order $\epsilon^{2}$ and higher. If mean field theory for the average mass distribution was exact, operator $\Pi$ would have been identically equal to zero. Consequently only loop diagrams contribute to $\Pi\left(t_{2}, t_{1}\right)$. The one-loop diagrams are shown in Fig. 6 (IIA). The computation of corresponding Feynman integrals is straightforward. After integration with respect to $t_{1}$ we find their respective contributions to $\Pi(t)$ :

$$
\begin{aligned}
\Pi_{(i)}(t) & =\frac{-8 \lambda t^{\epsilon / 2}}{(8 \pi)^{d / 2} t} \frac{(1+2 \epsilon)}{\epsilon(1+\epsilon / 2)^{2}}, \\
\Pi_{(i i)}(t) & =\frac{8 \lambda t^{\epsilon / 2}}{(8 \pi)^{d / 2} t} \frac{1}{\epsilon(1+\epsilon / 2)}, \\
\Pi_{(i i i)}(t) & =\frac{-8 \lambda t^{\epsilon / 2}}{(8 \pi)^{d / 2} t} \frac{1}{(1+\epsilon / 2)(2+\epsilon / 2)} .
\end{aligned}
$$

Note that individual contribution from diagrams (i) and (ii) are $1 / \epsilon$-times bigger than the contribution from diagram (iii), which does not contain primitive loops. Yet, terms of order $\epsilon^{-1}$ cancel upon adding $\Pi_{(i)}(t)$ and $\Pi_{(i i)}(t)$ leaving terms of order up to $\epsilon^{0}$ - same as the leading order of terms of $\Pi_{(i i i)}(t)$. This cancellation explains why we had to account for an apparently subleading contribution of diagram (iii) to the perturbative expansion of $\Pi(t)$. Such a cancellation is not accidental and happens at all orders of loop expansion: diagrams (i) and (ii) can be interpreted as first two terms in $\epsilon$-expansion of the first term in the cumulant expansion of $\Pi\left(t_{2}, t_{1}\right)$, see Fig. 6 (IIB). This term corresponds to an exact total particle density $\bar{N}(t)$ connected to the $\tilde{P} P$-propagator via an exact $\tilde{P} N P$-vertex. The vertex is of the order of the fixed point coupling $g^{*} \sim \epsilon$, while exact density is of the order $\epsilon^{-1}$, 19]. Therefore the contribution of the term in question to $\Pi\left(t_{2}, t_{1}\right)$ is of order 1 , which is reflected in cancellation of the terms of the lowest order in $\epsilon$ in every term of its loop expansion.
Adding together Eqs. (40), (41) and (42), we find that

$$
\Pi(t)=\frac{2 \lambda t^{\epsilon / 2}}{(8 \pi)^{d / 2} t}(1+O(\epsilon))+2 \text {-loop corrections. }
$$

Large-t behavior of $\Pi(t)$ can be obtained from Eq. (43) by replacing the 'bare' reaction rate $\lambda$ with renormalized reaction rate $\lambda_{R}(t) \sim 2 \pi \epsilon t^{-\epsilon / 2}, t \rightarrow \infty$. The result does indeed coincide with Eq. (37).

It remains to verify that two- and higher loop diagrams contribute only to higher order terms in the $\epsilon$-expansion of $\Pi(t)$. Order- $\epsilon^{-2}$ contributions from diagrams containing only primitive loops cancel as explained above. Order- $\epsilon^{-1}$ contributions from diagrams with two primitive loops and two-loop diagrams with one primitive loop are accounted for by one-loop renormalization of coupling constant in one-loop diagrams. Hence non-trivial corrections to polarization operator come only from twoloops diagrams containing no primitive loops and nonsingular parts of all other two-loop diagrams. Simple counting shows that contribution from such diagrams to $\Pi(t)$ is proportional to $\frac{\left(\lambda_{R}(t) t^{\epsilon / 2}\right)^{n}}{\epsilon^{(n-2)} t} \sim \frac{\epsilon^{2}}{t}$. Similar argument shows that $n$-loop diagrams contribute to $\Pi(t)$ at the order $\epsilon^{n}$ only.

Now it is very easy to characterize the class of diagrams giving the leading contribution to the $\epsilon$-expansion of $\Pi(t)$. The statistics of $N(t)$ is strongly non-Gaussian. Yet, the main contribution to the polarization operator $\Pi(t)$ comes from the diagrams proportional to the first and the second cumulants of the stochastic field $N(x, t)$ only. Non-Gaussian effects are due to the fact that these cumulants are connected to $\bar{P} P$-propagator via exact vertices.

It is also possible to derive formula Eq. (28) without using the formalism of renormalization group. Instead, one can solve equation Eq. (36) directly using one-loop expression for polarization operator $\Pi(t)$ in two dimensions. The latter can be obtained by setting in Eq. (43) $d=2$ and replacing the bare reaction rate $\lambda$ with renormalized reaction rate in two dimensions: $\lambda_{R}(t)=\frac{4 \pi}{\ln \left(t / t_{0}\right)}\left(1+O\left(1 / \ln \left(t / t_{0}\right)\right)\right)$. The presented expression for $\lambda_{R}(t)$ is easy to compute by explicit resummation of all diagrams contributing to renormalization of the bare reaction rate, see [19] for details. The resulting equation for $\eta(t) \equiv t^{2} P(t)$ is

$$
\dot{\eta}(t)=\frac{1}{t \ln \left(t / t_{0}\right)} \eta(t) .
$$

The solution is $\eta(t) \sim \ln \left(t / t_{0}\right)$. Correspondingly, $P(t) \sim$ $\frac{\ln \left(t / t_{0}\right)}{t^{2}}$, which coincides with Eq. (28).

\section{KANG-REDNER ANOMALY AND CORRECTIONS TO KOLMOGOROV PARTICLE SPECTRUM.}

Now we will show that the mean field result $\bar{P}(m, t) \sim$ $m^{0}$ can be interpreted as a constant flux (Kolmogorov) 
solution of the Smoluchowski equation. We will then interpret Kang-Redner anomaly as a breakdown of Kolmogorov scaling due to strong flux fluctuations developing at large times.

Averaging Eqs. (2) and (3) with respect to noise one gets the following relation between one- and two point mass distribution functions:

$$
\begin{aligned}
& \frac{\partial\langle P\rangle}{\partial t}=-\lambda\langle P N\rangle, \\
& \frac{\partial\langle N\rangle}{\partial t}=-\frac{1}{2} \lambda\left\langle N^{2}\right\rangle .
\end{aligned}
$$

Let us look for solutions of Eq. 46 having the form

$$
\bar{P}(m, t)=\frac{\bar{N}(t)}{M(t)} J(\mu, t),
$$

where $\mu=\frac{m}{M(t)}$. Recall that $M(t)=\bar{N}^{-1}$ is the typical mass. The new dependent variable $J$ has a simple physical meaning: $\int_{0}^{\mu} d \mu^{\prime} J\left(\mu^{\prime}, t\right)$ is the average number of particles with masses less than $M(t) \mu$ contained in the volume $\bar{N}^{-1}(t)$.

At times much less than $t_{c}=(\lambda)^{-2 / \epsilon}$ relative fluctuations of local density are small. As a result, mean field theory is applicable and $\langle J N\rangle \approx\langle J\rangle\langle N\rangle$. As a result, equation Eq. (45) simplifies to

$$
\frac{\partial}{\partial t}\left(\frac{J}{\mu}\right)=\frac{\partial J}{\partial \mu} .
$$

Therefore, $\frac{J}{\mu}$ is a locally conserved quantity with flux equal to $(-J)$. Note that $J>0$. Therefore the cascade of $\frac{J}{\mu}$ is inverse in the terminology of turbulence: its flux is directed towards the small masses. We see that selfsimilar solutions of Eq. (46) correspond to constant flux solutions of Eq. (48). The latter is just $J=$ Const. Constant flux solutions of kinetic equations are called Kolmogorov solutions in the theory of weak turbulence, see [23 for details. We therefore conclude that in the mean field approximation

$$
\langle P\rangle(m, t)=\frac{\bar{N}(t)}{M(t)} \mu^{e_{k o l m}},
$$

where $e_{\text {kolm }}=0$ is the exponent, which determines the Kolmogorov scaling of the average mass distribution. Note, that the flux $J$ also has a meaning of dimensionless particle density (the number of particles in the volume $\bar{N}^{-1}$, which is an obvious integral of motion.

The fact that $J=$ const, means that particles are equipartitioned between system's degrees of freedom and particles' flux is identically equal to zero. The characteristic feature of the state Eq. (49) of our system is therefore the presence of non-zero constant flux of one integral of motion and equipartition of the other. Similar kind of behavior has been observed in models of turbulent advection, 30]

We know however, that mean field approximation is invalid in the limit of large times if dimension is two or less because of strong fluctuations of local particle density . Using results of the previous sections one can interpret Kang-Redner anomaly as the anomaly in the constant flux condition:

$$
\mu \frac{\partial J}{\partial \mu}=\frac{e_{K R}}{d} J
$$

where $e_{K R}$ is Kang-Redner's exponent.

Solving Eq. (50), we find that $P(m, t) \sim \mu^{e}$, where $e=$ $e_{k o l m}+\frac{e_{K R}}{d}$. Therefore, Kang-Redner anomaly can be also interpreted as a correction to Kolmogorov scaling of the average mass distribution due to strong fluctuations.

\section{CONCLUSION.}

In the present paper we have shown that the problem of cluster-cluster aggregation in $d \leq 2$ can be effectively analysed using renormalization group method. We have demonstrated that the dependence of average mass distribution on mass is determined by the anomalous dimension of the stochastic field $P$ (local mass distribution). This anomaly is due to the relevance of 'boundary' $(t=0)$ fluctuations for the large times asymptotics of $\bar{P}(m, t)$. In that respect the phenomenon of KangRedner anomaly resembles the phenomenon of boundary phase transition in equilibrium statistical mechanics, see [2] for a review. Formally, the anomalous dimension of the local mass distribution is a consequence of the nontriviality of the $\gamma$-function of the effective field theory Eq. (12). The fact that $\gamma(g) \neq 0$ is ultimately responsible for the breakdown of the Smoluchowski theory (or equivalently, the renormalized mean field theory) applied to the model at hand. At present, renormalization group analysis seems to be the only theoretical method of studying the problem of cluster-cluster aggregation in $d>1$. This is not quite satisfactory, as the analysis is essentially perturbative in nature. However, our theoretical predictions concerning the behavior of the average mass distributions at small masses in two dimensions have been unambiguously confirmed numerically.

In section V1 we have shown that there is a relation between cluster-cluster aggregation and the theory of weak turbulence. In particular, we demonstrated that Kang-Redner anomaly can be interpreted as a correction to the Kolmogorov spectrum of particles in the mass space. However, we must stress that the analogy between our model and the phenomenon of turbulence must be taken with a pinch of salt: while the cascade of the conserved quantity in our model happens along the mass axis only, conserved quantities (such as energy or enstrophy) in more traditional turbulent systems flow through the scales of the physical space.

The method of dynamical renormalization group developed in the context of the model at hand can be applied to other non-equilibrium particle systems as well. In particular the problem of cluster-cluster aggregation 
with annihilation of particles, can be solved using techniques similar to those outlined in this paper [31]. This latter problem is related to the computation of the domain wall persistence exponent for the 1d q-state Potts model 31.

\section{ACKNOWLEDGMENTS.}

The authors would like to thank John Cardy for many insights into the applications of renormalization group.
Useful discussions with Robin Ball, Colm Connaughton, Igor Kolokolov, Anttii Kupiainen, Vladimir Lebedev, Paolo Muratore-Ginanneschi, Sergey Nazarenko are also appreciated. OZ would like to acknowledge the hospitality of the Department of Mathematics of Helsinki University where part of the present work was done. The work at Oxford was supported by EPSRC, UK.
[1] V. Privman, Trends in Statistical Phys. 1, 89 (1994).

[2] J. Cardy, Scaling and Renormalization in Statistical Physics, Cambridge University Press, (1996).

[3] K. Kang and S. Redner, Phys. Rev. A 30, 2833 (1984).

[4] J. L. Spouge, Phys. Rev. Lett. 60, 871 (1988).

[5] S. N. Majumdar and D. Huse, Phys. Rev. E 52, 270 (1995).

[6] O. Zaboronski, Phys. Letts. A 281, 119 (2001).

[7] D. J. Aldous, Bernoulli 5, 3 (1999).

[8] G. Oshanin and M. Moreau, J. Chem. Phys. 102, 2977 (1995).

[9] E. K. O. Hellén, P. E. Salmi and M. J. Alava, Europhys. Lett. 59, 186 (2002).

[10] D. ben-Avraham in Nonequilibrium Statistical Mechanics in One Dimension ed. V. Privman (Cambridge Univ. Press, Cambridge, 1997).

[11] W. H. White, J. Colloid Interface Sci. 87, 204 (1982).

[12] S. K. Friedlander, Smoke, Dust and Haze (Wiley Interscience, New York, 1977).

[13] A. E. Scheidegger, Bull. I.A.S.H. 12, 15 (1967).

[14] W. R. White and P. Wiltzius, Phys. Rev. Lett. 75, 3012 (1995).

[15] J. Cardy, Field theory and nonequilibrium statistical mechanics. The notes are available at the website http: //www.thphys.physics.ox.ac.uk/users/JohnCardy/.

[16] A. S. Mikhailov, Phys. Lett. A 85, 214 (1981); Phys. Lett A 85, 427 (1981).

[17] L. Peliti, J. Phys. A 19, L365 (1986).

[18] P.-A. Ray and M. Droz, J. Phys. A 30, 1101 (1997).

[19] B. P. Lee, J. Phys. A 27, 2633 (1994).

[20] B. Lee, J. Cardy, J. Stat. Phys. 80, 971 (1995).

[21] P. Krapivsky, E. Ben-Naim and S. Redner, Phys. Rev. E
50, 2474 (1994).

[22] P. L. Krapivsky, Phys. Rev. E 49, 3233 (1994).

[23] V. E. Zakharov, V. S. L'vov and G. Falkovich, Kolmogorov Spectra of Turbulence I, Springer-Verlag Berlin Heidelberg (1992).

[24] M. Doi, J. Phys. A 9, 1465 (1976); M. Doi, J. Phys. A 9, 1479 (1976); Ya. B. Zel'dovich and A. A. Ovchinnikov, Zh. Eksp. Teor. Fiz. 78, 1588 (1978).

[25] M. Fisher, J. Stat. Phys. 34 , 667 (1984)

[26] The statement of correctness of mean field theory in $d>2$ has to be understood properly. For instance, the effective reaction rate entering the Smoluchowski coagulation equation is not equal to the bare reaction rate due to strong ultraviolet divergence of Feynman integrals contributing to renormalization of the reaction rate. As a result, density-density correlation function is not equal to the product of one-point function, but rather is proportional to it. Yet, as the renormalized reaction rate in $d>2$ is a constant depending on lattice spacing, decay exponents are predicted by the mean field correctly.

[27] P. C. Martin, E. D. Siggia and H. H. Rose, Phys. Rev. A 8, 423 (1973).

[28] C. Itzykson and J.-M. Drouffe, Statistical Field Theory, Cambridge monographs on mathematical physics, Cambridge University Press (1994).

[29] M. Howard, J. Phys. A 29, 3437 (1996). Cambridge University Press (2001).

[30] J.-P. Laval, S. Nazarenko, Journal of Fluid Mech. 408, 301 (2000).

[31] S. Krishnamurthy, R. Rajesh and O. Zaboronski, in preparation. 